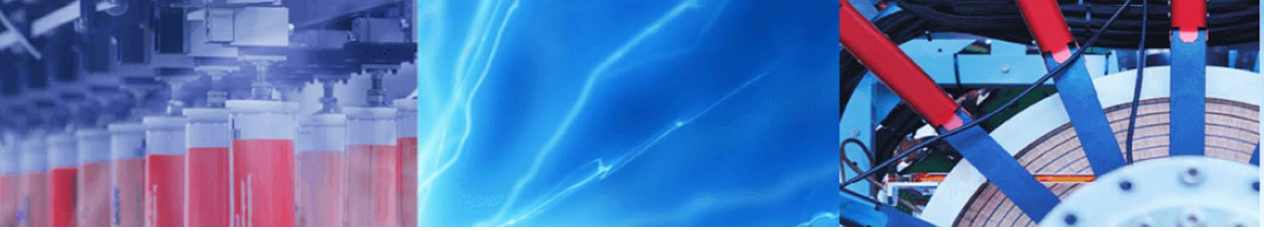

Research Article

\title{
Solving for the random component time-fractional partial differential equations with the new Sumudu transform iterative method
}

\author{
Halil Anaç ${ }^{1}$ (D) Mehmet Merdan ${ }^{1}$ (D) - Tülay Kesemen² ${ }^{2}$
}

Received: 2 November 2019 / Accepted: 30 March 2020 / Published online: 22 May 2020

(c) Springer Nature Switzerland AG 2020

\begin{abstract}
The new Sumudu transform iterative method is implemented to get the approximate solutions of random component time-fractional partial differential equations with Caputo derivative. The parameters and the initial conditions of the random component time-fractional partial differential equations are analyzed with Gamma distribution. The expected values and variances of these solutions are calculated, and the graphs of the expected values and variances are plotted in Maple software. The results for the random component time-fractional partial differential equations with Gamma distribution are examined to investigate effects of this distribution on results. The numerical experiments indicate that this method is very effective.
\end{abstract}

Keywords Sumudu transform · Gamma disribution · Caputo fractional derivative · Mittag-Leffler function

\section{Introduction}

Fractional calculus is a considerably important topic in several scientific areas [1-7]. Methodologies of fractional calculus have been widely used in the modeling of many real matters in applied mathematics.

Specifically, fractional partial differential equations (FPDEs) describe certain many phenomena in various areas of science and engineering such as damping laws, diffusion equations, heat transfer modeling, electrostatics, electrodynamics, fluid flow, elasticity and many others [8-18].

In the literature, there are very few studies on random fractional partial differential equations (RFPDEs). Random fractional partial differential equations (RFPDEs) are defined as fractional partial differential equations with random inputs that can be a random variable or a stochastic process. These equations have a great importance in many applications in areas such as engineering, biology, physics, mathematics and many other applied sciences.

It is almost impossible to solve random nonlinear fractional partial differential equations analytically. So various numerical methods and approximation schemes for RFPDEs, ordinary differential equations and fractional partial differential equations have been developed. There are many numerical methods and approximation schemes as adomian decomposition method (ADM) [19], homotopy perturbation method [20], collocation method [21-26], Galerkin-type method [27], differential transformation method (DTM) [28], variational iteration method (VIM) [29], fractional variational iteration method (FVIM) [30], random finite difference scheme [31, 32] and many other methods. The key motivation of writing this paper is to analyze random nonlinear fractional partial differential equations using the new Sumudu transform iterative method (NSTIM) which is the reliable computational method.

\footnotetext{
$\triangle$ Halil Anaç, halilanac@gumushane.edu.tr; Mehmet Merdan, mehmetmerdan@gmail.com; Tülay Kesemen, tkesemen@gmail.com | ${ }^{1}$ Department of Mathematical Engineering, Gumushane University, 29100 Gumushane, Turkey. ${ }^{2}$ Department of Mathematics, Karadeniz Technical University, Trabzon, Turkey.
} 
This paper studies random component time-fractional partial differential equations and to solve numerically by the new Sumudu transform iterative method (NSTIM). Wang and Liu [33] established this new Sumudu transform iterative method (NSTIM). They succesfully applied this method to obtain the approximate analytical solutions of time-fractional Cauchy reaction-diffusion equations.

In the literature, there are very few research work and articles on the power series transformation such as Sumudu transform. The Sumudu transform method (STM), proposed by Watugala, was applied to solve engineering problems [34]. The method was applied to partial differential equations by Weerakoon [35]. Then, Weerakoon found the inverse formula of this transform [36]. Demiray et al. [37] used the Sumudu transform method (STM) to get exact solutions of fractional differential equations. Kumar and Daftardar-Gejji [38] extended Sumudu transform iterative method (STIM) to solve a variety of time and space FPDEs and systems of FPDEs. Rahman and Ahmad applied fuzzy Sumudu transform (FST) for solving linear fuzzy fractional differential equations (FFDEs) by Caputo fuzzy fractional derivative [39]. Prakash et al [40] suggested a new iterative Sumudu transform method (NISTM) to solve numerically nonlinear time fractional Zahkarov-Kuznetsov equations.

In this paper, approximate solutions of two random component time-fractional partial differential equations are obtained by the new Sumudu transform iterative method (NSTIM) and variational iteration method. Projected technique is used for error analysis. In addition, approximate solutions obtained with two methods and exact solutions are shown in comparison tables. The difference of this work from Prakash et al. [40] and Wang and Liu [33] studies is to examine the random component fractional partial differential equations.

The aim of this study is to present the application of the new Sumudu transform iterative method for obtaining the approximate analytical solutions of random component time-fractional partial differential equations with Caputo derivative and for calculating the expected values and variances of these solutions. It is observed that the numerical solutions obtained by NSTIM for RFPDEs are almost similar to exact solutions for RFPDEs. Tables show that absolute errors are negligible. It is observed that NSTIM is superior than VIM for RFPDEs.

\section{Basic definitions}

A few basic definitions and properties of fractional calculus, Sumudu transform and Gamma distribution are presented in this section.
Definition $2.1[33,41]$ A real function $f(x), x>0$, is said to be in the space $C_{\mu^{\prime}} \mu \in \mathbb{R}$ if there exists a real number $p,(p>\mu)$, such that $f(x)=x^{p} f_{1}(x)$, where $f_{1}(x) \in C[0, \infty)$, and it is said to be in the space $C_{\mu}^{m}$ if $f^{(m)} \in C_{\mu}, m \in N$.

Definition $2.2[1,33,42]$ The Riemann-Liouville fractional integral operator of order $a \geq 0$, of a function $f \in C_{\mu}, \mu \geq-1$ is given in the following

$I^{a} f(x)= \begin{cases}\frac{1}{\Gamma(a)} \int_{0}^{x}(x-t)^{a-1} f(t) \mathrm{d} t, & a>0, x>0, \\ l^{0} f(x)=f(x), & a=0\end{cases}$

where $\Gamma($.$) is the Gamma function.$

Some features of the operator $I^{a}$ that will be used in this study are given below [33]:For $f \in C_{\mu}, \mu, \gamma \geq-1, \alpha, \beta \geq 0$,

1. $\left.\left.\right|^{a}\right|^{\beta} f(x)=\left.\left.\right|^{\beta}\right|^{a} f(x)=\left.\right|^{a+\beta} f(x)$,

2. $I^{a} X^{\gamma}=\frac{\Gamma(\gamma+1)}{\Gamma(\alpha+\gamma+1)} x^{\alpha+\gamma}$.

Definition $2.3[1,7,33,42]$ The Caputo fractional derivative of $f(x)$ is defined by

$D^{a} f(x)=l^{a-n} D^{n} f(x)=\frac{1}{\Gamma(n-a)} \int_{0}^{x}(x-t)^{n-a-1} f^{(n)}(t) \mathrm{d} t$

where $n-1\langle a \leq n, n \in N, x\rangle 0, f \in C_{-1}^{n}$.

Some properties of the operator $D^{a}$ that will be used in this study are given below [33]:

3. $D^{a} l^{a} f(x)=f(x)$,

4. $I^{a} D^{a} f(x)=f(x)-\sum_{k=0}^{n-1} f^{(k)}\left(0^{+}\right) \frac{x^{k}}{k !}, x>0$.

Definition $2.4[33,43]$ For $a>0$, the Mittag-Leffler function $E_{a}$ is given as follows

$E_{a}(z)=\sum_{n=0}^{\infty} \frac{z^{a}}{\Gamma(n a+1)}$.

Definition 2.5 $[33,43]$ The Sumudu transform on the set of functions $A=\left\{f(t)\left|\exists M, \tau_{1}, \tau_{2}>0,\right| f(t) \mid<M e^{\frac{t}{\tau_{j}}}\right.$, ift $\left.\in(-1)^{j} \times[0, \infty)\right\}$ is given as follows

$S[f(t)]=\int_{0}^{\infty} e^{-t} f(v t) \mathrm{d} t, \quad v \in\left(\tau_{1}, \tau_{2}\right)$. 
Definition 2.6 [33] The Sumudu transform of the Caputo fractional derivative with respect to $x$ is given below

$$
\begin{aligned}
S\left[D_{x}^{n a} u(x, t)\right] & =v^{-n a} S[u(x, t)] \\
& -\sum_{k=0}^{n-1} v^{(-n a+k)} u^{(k)}(0, t), \quad n-1<n a \leq n .
\end{aligned}
$$

Definition 2.7 [44] If the probability density function of a random variable $X$ has the following form, this random variable has the Gamma distribution and is called a Gamma random variable. For $x, \alpha, \beta>0$,

$f(x)=\frac{x^{\alpha-1} e^{-x / \beta}}{\Gamma(\alpha) \beta^{\alpha}}$

If the random variable $X$ has a Gamma distribution with parameters $\alpha$ and $\beta$, the expected value and variance of the random variable $X$ are given as follows

$E(X)=\alpha \beta, \quad \operatorname{Var}(X)=\alpha \beta^{2}$.

In this study, Gamma distribution in the examples is chosen as Gamma ( $\alpha=3, \beta=2)$.

Theorem 2.1 [40] A real number $0<k<1$ satisfies $u_{m+1}(x, t) \leq k u_{m}(x, t)$ for all values of $m$. Furthermore, if the truncated series $\sum_{m=0}^{l} u_{m}(x, t)$ is used as an approximate solution $u(x, t)$, then maximum absolute truncated error is obtained by

$\left\|u(x, t)-\sum_{m=0}^{l} u_{m}(x, t)\right\| \leq \frac{k^{l+1}}{(1-k)}\left\|u_{0}(x, t)\right\|$.

\section{The new Sumudu transform iterative method}

Consider the following equation with the initial condition

$$
\left\{\begin{array}{l}
D_{t}^{n a} u(x, t)+L u(x, t)+R u(x, t)=g(x, t), \\
n-1<n a \leq n, \\
u(x, 0)=h(x) .
\end{array}\right.
$$

where $D_{t}^{n a}$ is the Caputo fractional derivative operator, $D_{t}^{n a}=\frac{\partial^{\text {nt }}}{\partial t^{\text {na }}}, g(x, t)$ is a continuous function, and $L$ and $R$ are linear and nonlinear operators, respectively [33].

If Sumudu transform is implemented to both sides of Eq. (8), it can be found that

$S\left[D_{t}^{n a} u(x, t)+L u(x, t)+R u(x, t)\right]=S[g(x, t)]$.

The following equation is obtained with the feature of the Sumudu transform [33]
$S[u(x, t)]-v^{n a} \sum_{k=0}^{n-1} u^{(k)}(x, 0)+v^{n a} S[L u(x, t)+R u(x, t)-g(x, t)]=0$.

From Eq. (10), it can be found that

$S[u(x, t)]=v^{n a} \sum_{k=0}^{n-1} u^{(k)}(x, 0)-v^{n a} S[L u(x, t)+R u(x, t)-g(x, t)]$.

If the inverse Sumudu transform is applied to Eqs. (11) and (12) is obtained

$$
\begin{aligned}
u(x, t)= & S^{-1}\left[v^{n a} \sum_{k=0}^{n-1} u^{(k)}(x, 0)\right] \\
& -S^{-1}\left[v^{n a} S[\operatorname{Lu}(x, t)+R u(x, t)-g(x, t)]\right] .
\end{aligned}
$$

Wang and Liu [33] assumed that the following equalities are hold:

$\left\{\begin{array}{l}f(x, t)=S^{-1}\left[v^{n a} \sum_{k=0}^{n-1} u^{(k)}(x, 0)+v^{n a} S[g(x, t)]\right], \\ N(u(x, t))=-S^{-1}\left[v^{n a} S[R u(x, t)]\right], \\ K(u(x, t))=-S^{-1}\left[v^{n a} S[\operatorname{Lu}(x, t)]\right] .\end{array}\right.$

So Eq. (12) becomes Eq. (13):

$u(x, t)=f(x, t)+K(u(x, t))+N(u(x, t))$,

where $f$ is a known function, $K$ is a linear operator of $u$ and $N$ is a nonlinear operator of $u$. The solution of Eq. (13) is given by the series form as follows [33]

$u(x, t)=\sum_{i=0}^{\infty} u_{i}(x, t)$

Since $\mathrm{K}$ is a linear operator, the following equation can be written as

$$
K\left(\sum_{i=0}^{\infty} u_{i}\right)=\sum_{i=0}^{\infty} K\left(u_{i}\right) .
$$

The nonlinear operator $N$ can be written as $[33,45]$

$$
N\left(\sum_{i=0}^{\infty} u_{i}\right)=N\left(u_{0}\right)+\sum_{i=0}^{\infty}\left[N\left(\sum_{j=0}^{i} u_{j}\right)-N\left(\sum_{j=0}^{i-1} u_{j}\right)\right] .
$$

Thus, Eq. (13) is given as [33]

$$
\sum_{i=0}^{\infty} u_{i}=f+\sum_{i=0}^{\infty} K\left(u_{i}\right)+N\left(u_{0}\right)+\sum_{i=0}^{\infty}\left[N\left(\sum_{j=0}^{i} u_{j}\right)-N\left(\sum_{j=0}^{i-1} u_{j}\right)\right] .
$$


If the following recurrence is defined

$\left\{\begin{array}{l}u_{0}=f \\ u_{1}=K\left(u_{0}\right)+N\left(u_{0}\right), \\ u_{m+1}=K\left(u_{m}\right)+N\left(u_{0}+\cdots+u_{m}\right)-N\left(u_{0}+u_{1}+\cdots+u_{m-1}\right) .\end{array}\right.$

the Eq. (19) is obtained [33]

$\left(u_{1}+\cdots+u_{m+1}\right)=K\left(u_{0}+\cdots+u_{m}\right)+N\left(u_{0}+\cdots+u_{m}\right)$.

Thus, Eq. (20) is obtained as

$\sum_{i=0}^{\infty} u_{i}=f+K\left(\sum_{i=0}^{\infty} u_{i}\right)+N\left(\sum_{i=0}^{\infty} u_{i}\right)$.

The $m$-term approximate solution of Eq. (13) is obtained as

$u=u_{0}+u_{1}+u_{2}+u_{3}+\cdots+u_{m-1}$.

In this work, we obtain the approximate analytical solutions of random component time-fractional partial differential equations with the new Sumudu transform iterative method. It is illustrated in the numerical experiments.

\section{Numerical experiments}

\section{Example 1 Consider the RFPDE}

$$
\left\{\begin{array}{l}
u_{t}^{\alpha}(x, t)+u(x, t) u_{x}(x, t)+u(x, t)(1+u(x, t))=0 \\
0<\alpha \leq 1 \\
u(x, 0)=A e^{-x}
\end{array}\right.
$$

where $A$ is a Gamma distributed random variable with parameters $\alpha=3$ and $\beta=2$, i.e., $A \sim G(\alpha=3, \beta=2)$.

If Sumudu transform is applied to Eq. (22) and the differential feature of Sumudu transform is used, Eq. (23) is found

$$
S[u]=u(x, 0)+v^{\alpha} S\left[-u u_{x}-u-u^{2}\right] .
$$

If the inverse Sumudu transform is applied to Eqs. (23) and (24) is obtained

$$
u(x, t)=S^{-1}\left[A e^{-x}\right]+S^{-1}\left[v^{\alpha} S\left[-u u_{x}-u-u^{2}\right]\right] .
$$

From Eq. (24), it is obtained as

$$
u(x, t)=A e^{-x}+S^{-1}\left[\nu^{\alpha} S\left[-u u_{x}-u-u^{2}\right]\right] .
$$

For NSTIM, Eq. (26) holds:
$\left\{\begin{array}{l}u_{0}=A e^{-x}, \\ K[u(x, t)]=S^{-1}\left[v^{\alpha} S\left[-u u_{x}-u-u^{2}\right]\right] .\end{array}\right.$

By iteration, the results are obtained as follows

$u_{0}=A e^{-x}$,

$u_{1}=\frac{-A e^{-x} t^{\alpha}}{\Gamma(1+\alpha)}$

$u_{2}=\frac{A e^{-x} t^{2 \alpha}}{\Gamma(1+2 \alpha)}$,

$u_{3}=\frac{-A e^{-x} t^{3 \alpha}}{\Gamma(1+3 \alpha)}$

$\vdots$

$u_{n}=\frac{(-1)^{n} A e^{-x} t^{n \alpha}}{\Gamma(1+n \alpha)}=\frac{A e^{-x}(-t)^{n \alpha}}{\Gamma(1+n \alpha)}$.

Thus, the approximate solution of Eq. (22) is obtained as the following

$$
\begin{aligned}
u_{a}(x, t) & =A e^{-x}-\frac{A e^{-x} t^{\alpha}}{\Gamma(1+\alpha)}+\frac{A e^{-x} t^{2 \alpha}}{\Gamma(1+2 \alpha)}-\frac{A e^{-x} t^{3 \alpha}}{\Gamma(1+3 \alpha)}+\cdots+\frac{A e^{-x}(-t)^{n \alpha}}{\Gamma(1+n \alpha)} \\
& =A e^{-x}\left(1-\frac{t^{\alpha}}{\Gamma(1+\alpha)}+\frac{t^{2 \alpha}}{\Gamma(1+2 \alpha)}-\frac{t^{3 \alpha}}{\Gamma(1+3 \alpha)}+\cdots+\frac{(-t)^{n \alpha}}{\Gamma(1+n \alpha)}\right) \\
& =A e^{-x} E_{\alpha}\left[(-t)^{\alpha}\right] .
\end{aligned}
$$

The form $u_{a}(x, t)=A e^{-x-t}$ is the approximate solution of the Eq. (22) for $a=1$. Also, this form is the exact solution of the Eq. (22) for $\alpha=1$.

In Table 1, we chose $a=1$, because for $a=1$, it allows us to compare the the exact solution between approximate solution obtained with the fifth-order NSTIM and the VIM solution. $A=6$ is randomly chosen. So the approximate solution from fifth-order NSTIM indicates that it is closer to the exact solution than the approximate solution from VIM for a random $A$ in Example 1. It provided a comparison of the fifth-order NSTIM and VIM solution for $a=0.9$ and $A=6$ in Table 2.

It is observed from Table 1 that the numerical solution obtained by NSTIM is almost similar to exact solution. Table 3 shows that absolute error is negligible, because the absolute errors in Table 1 of the article of Sakar and Ergören are bigger than our absolute errors [46]. Table 3 shows the comparison of absolute error between approximate solutions acquired from different methods and exact solution different values of $x$ and $t$. It is observed from Tables 1,2 and 3 that NSTIM is superior than VIM.

Let $A \sim G(\alpha=3, \beta=2)$.

So the approximated values of $A$ and $A^{2}$ are obtained as follows

$E(A)=3.2=6, E\left(A^{2}\right)=3.4(1+3)=48$.

The approximated value of Eq. (27) is given by 
Table 1 Comparison of the exact solution, approximate solution obtained with the fifth-order NSTIM and the VIM solution for $\alpha=1$ and $A=6$

\begin{tabular}{lllll}
\hline$x$ & $t$ & Exact sol. & NSTIM & VIM \\
\hline 0.5 & 0.2 & 2.97951181 & 2.97951150 & 2.98413084 \\
0.5 & 0.4 & 2.43941795 & 2.43939838 & 2.47464509 \\
0.5 & 0.6 & 1.99722650 & 1.99700947 & 2.11072669 \\
0.5 & 0.8 & 1.63519075 & 1.63400330 & 1.89237565 \\
0.5 & 1.0 & 1.33878096 & 1.33436745 & 1.81959197 \\
1.0 & 0.2 & 1.80716527 & 1.80716508 & 1.80996685 \\
1.0 & 0.4 & 1.47958178 & 1.47956991 & 1.50094812 \\
1.0 & 0.6 & 1.21137910 & 1.21124857 & 1.28022045 \\
1.0 & 0.8 & 0.99179332 & 0.99107310 & 1.14778385 \\
1.0 & 1.0 & 0.81201169 & 0.80933477 & 1.10363832 \\
1.5 & 0.2 & 1.09610114 & 1.09610102 & 1.09780038 \\
1.5 & 0.4 & 0.89741171 & 0.89740451 & 0.91037105 \\
1.5 & 0.6 & 0.73473856 & 0.73465872 & 0.77649295 \\
1.5 & 0.8 & 0.60155306 & 0.60111622 & 0.69616609 \\
1.5 & 1.0 & 0.49250999 & 0.49088635 & 0.66939048 \\
\hline
\end{tabular}

Table 2 Comparison of the fifth-order NSTIM and VIM solution for $\alpha=0.9$ and $A=6$.

\begin{tabular}{llll}
\hline$x$ & $t$ & NSTIM & VIM \\
\hline 0.5 & 0.2 & 2.85953155 & 2.84840575 \\
0.5 & 0.4 & 2.33297833 & 2.28819073 \\
0.5 & 0.6 & 1.93196722 & 1.90982510 \\
0.5 & 0.8 & 1.61509200 & 1.70165945 \\
0.5 & 1.0 & 1.35590765 & 1.65793613 \\
1.0 & 0.2 & 1.73439356 & 1.72764542 \\
1.0 & 0.4 & 1.41502288 & 1.38785783 \\
1.0 & 0.6 & 1.17179735 & 1.15836748 \\
1.0 & 0.8 & 0.97960282 & 1.03210863 \\
1.0 & 1.0 & 0.82239956 & 1.00558910 \\
1.5 & 0.2 & 1.05196287 & 1.04786991 \\
1.5 & 0.4 & 0.85825476 & 0.84177832 \\
1.5 & 0.6 & 0.71073102 & 0.70258539 \\
1.5 & 0.8 & 0.59415914 & 0.62600552 \\
1.5 & 1.0 & 0.49881054 & 0.60992062 \\
\hline
\end{tabular}

$E\left[u_{a}(x, t)\right]=E[A] e^{-x} E_{\alpha}\left[(-t)^{\alpha}\right]=6 e^{-x} E_{\alpha}\left[(-t)^{\alpha}\right]$.

The graphs of the approximated values of the Eq. (27) for different $\alpha$ values are plotted in Maple software as follows (Figs. 1, 2, 3, 4).

For $A \sim G(\alpha=3, \beta=2)$, the variance of Eq. (27) is calculated as follows

$$
\begin{aligned}
V\left[u_{a}(x, t)\right] & =E\left[u^{2}(x, t)\right]-(E[u(x, t)])^{2} \\
& =E\left[A^{2}\right] e^{-2 x}\left(E_{\alpha}\left[(-t)^{\alpha}\right]\right)^{2}-(E[A])^{2} e^{-2 x}\left(E_{\alpha}\left[(-t)^{\alpha}\right]\right)^{2} \\
& =48 e^{-2 x}\left(E_{\alpha}\left[(-t)^{\alpha}\right]\right)^{2}-36 e^{-2 x}\left(E_{\alpha}\left[(-t)^{\alpha}\right]\right)^{2} \\
& =12 e^{-2 x}\left(E_{\alpha}\left[(-t)^{\alpha}\right]\right)^{2} .
\end{aligned}
$$

The graphs of the variances of the Eq. (27) for different $\alpha$ values are plotted in Maple software as follows (Figs. 5, $6,7,8)$

Example 2 Consider the RFPDE

$$
\left\{\begin{array}{l}
u_{t}^{\alpha}(x, t)+u(x, t) u_{x}(x, t)+u(x, t)(1+u(x, t)) \ln a=0 \\
0<\alpha \leq 1 \\
u(x, 0)=A a^{-x}
\end{array}\right.
$$

where $A$ is a Gamma distributed random variable with parameters $\alpha=3$ and $\beta=2$, i.e., $A \sim G(\alpha=3, \beta=2)$.

If Sumudu transform is applied to Eq. (28) and the differential feature of Sumudu transform is used, Eq. (29) is found

$S[u]=u(x, 0)+v^{\alpha} S\left[-u u_{x}-u \ln a-u^{2} \ln a\right]$.

If the inverse Sumudu transform is applied to Eqs. (29) and (30) is obtained

$u(x, t)=S^{-1}\left[A a^{-x}\right]+S^{-1}\left[v^{\alpha} S\left[-u u_{x}-u \ln a-u^{2} \ln a\right]\right]$.

From Eq. (30), it is obtained as

$u(x, t)=A a^{-x}+S^{-1}\left[v^{\alpha} S\left[-u u_{x}-u \ln a-u^{2} \ln a\right]\right]$.

For NSTIM, Eq. (32) holds:

$\left\{\begin{array}{l}u_{0}=A a^{-x}, \\ K[u(x, t)]=S^{-1}\left[v^{\alpha} S\left[-u u_{x}-u \ln a-u^{2} \ln a\right]\right] .\end{array}\right.$

By iteration, the results are obtained as follows

$$
\begin{aligned}
u_{0} & =A a^{-x}, \\
u_{1} & =\frac{-A a^{-x} t^{\alpha} \ln a}{\Gamma(1+\alpha)}, \\
u_{2} & =\frac{A a^{-x} t^{2 \alpha} \ln ^{2} a}{\Gamma(1+2 \alpha)}, \\
u_{3} & =\frac{-A a^{-x} t^{3 \alpha} \ln ^{3} a}{\Gamma(1+3 \alpha)}, \\
& \vdots \\
u_{n} & =\frac{A a^{-x}\left(-\ln a t^{\alpha}\right)^{n}}{\Gamma(1+n \alpha)} .
\end{aligned}
$$


Table 3 Comparison between absolute error when $\alpha=1$ and $A=6$, for Example 1

\begin{tabular}{|c|c|c|c|c|c|c|c|}
\hline & \multirow[t]{2}{*}{$x$} & \multicolumn{6}{|l|}{$t$} \\
\hline & & 0.00 & 0.02 & 0.04 & 0.06 & 0.08 & 0.10 \\
\hline NSTIM & 0.00 & 0.00000 & 0.00000000 & 0.00000000 & 0.00000003 & 0.00000016 & 0.00000049 \\
\hline VIM & & 0.00000 & 0.00000790 & 0.00006300 & 0.00021000 & 0.00050000 & 0.00097000 \\
\hline NSTIM & 0.02 & 0.00000 & 0.00000000 & 0.00000000 & 0.00000003 & 0.00000015 & 0.00000048 \\
\hline VIM & & 0.00000 & 0.00000780 & 0.00006200 & 0.00020000 & 0.00049000 & 0.00095000 \\
\hline NSTIM & 0.04 & 0.00000 & 0.00000000 & 0.00000000 & 0.00000003 & 0.00000015 & 0.00000047 \\
\hline VIM & & 0.00000 & 0.00000760 & 0.00006000 & 0.00020000 & 0.00048000 & 0.00093000 \\
\hline NSTIM & 0.06 & 0.00000 & 0.00000000 & 0.00000000 & 0.00000003 & 0.00000015 & 0.00000046 \\
\hline VIM & & 0.00000 & 0.00000740 & 0.00005900 & 0.00020000 & 0.00047000 & 0.00091000 \\
\hline NSTIM & 0.08 & 0.00000 & 0.00000000 & 0.00000000 & 0.00000003 & 0.00000014 & 0.00000045 \\
\hline VIM & & 0.00000 & 0.00000730 & 0.00005800 & 0.00019000 & 0.00046000 & 0.00090000 \\
\hline NSTIM & 0.10 & 0.00000 & 0.00000000 & 0.00000000 & 0.00000003 & 0.00000014 & 0.00000044 \\
\hline VIM & & 0.00000 & 0.00000720 & 0.00005700 & 0.00019000 & 0.00045000 & 0.00088000 \\
\hline
\end{tabular}

$E(u a(x, t))$

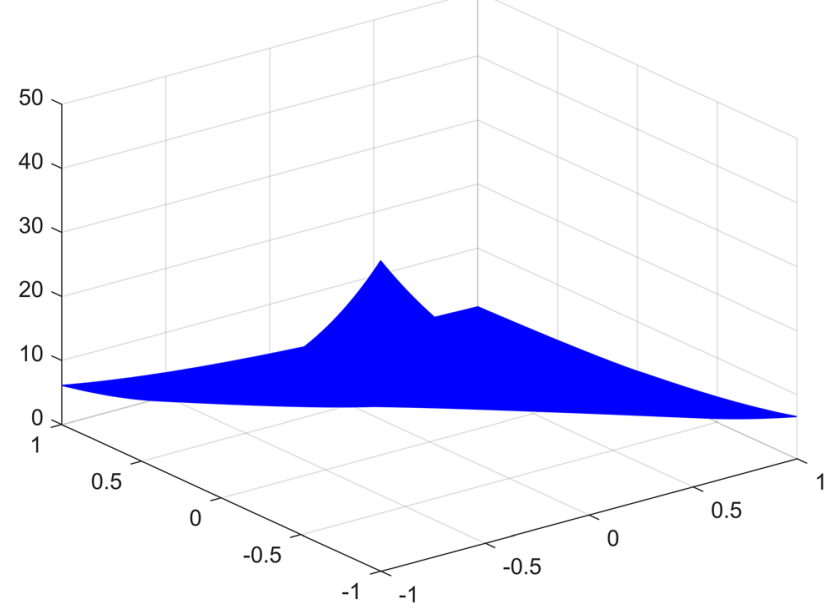

Fig. 1 For $\alpha=0.9$, time-dependent variation of approximated value of the Eq. (22)

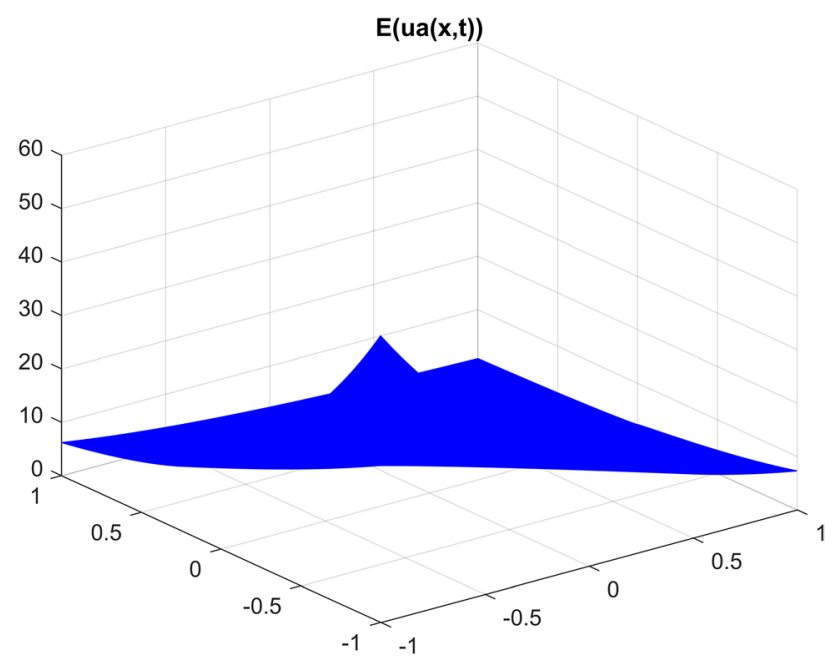

Fig. 2 For $\alpha=0.8$, time-dependent variation of approximated value of the Eq. (22)
$E(u a(x, t))$

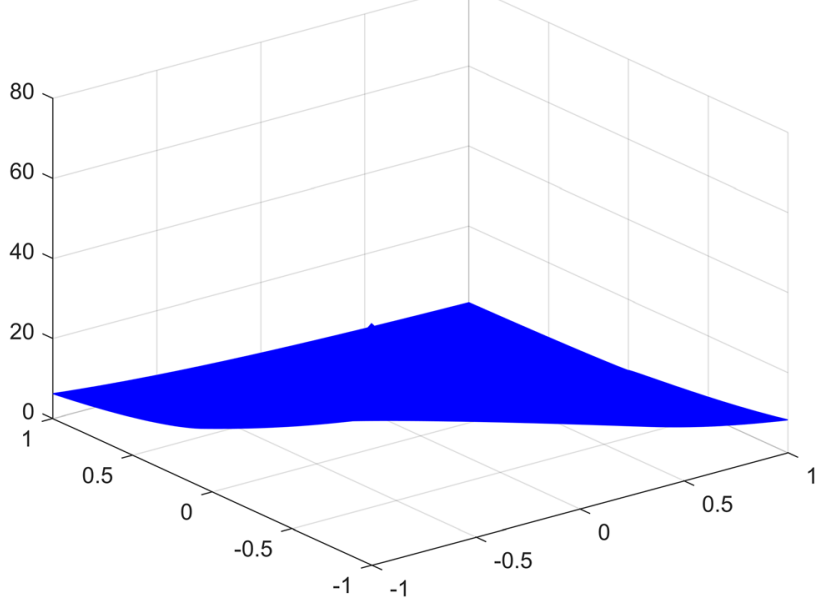

Fig. 3 For $\alpha=0.7$, time-dependent variation of approximated value of the Eq. (22)

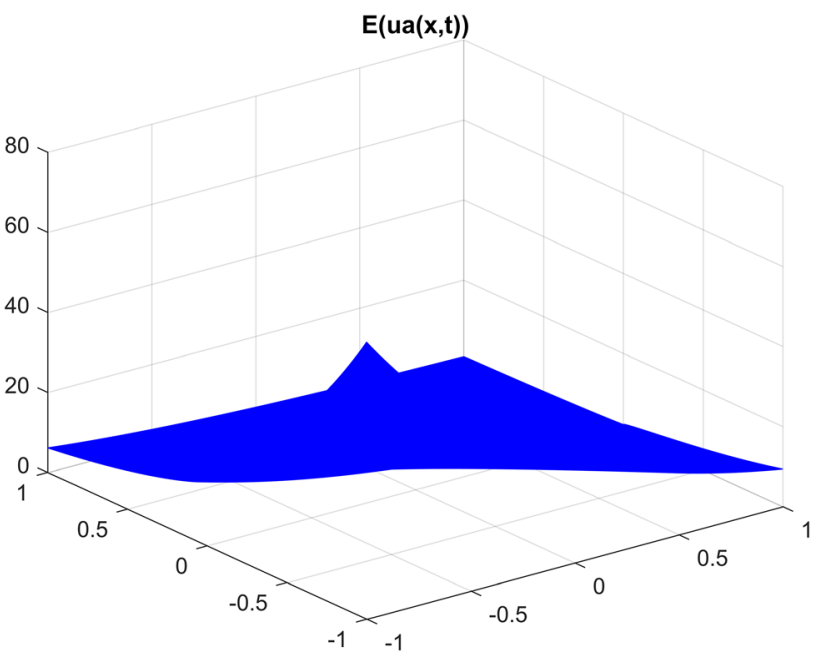

Fig. 4 For $\alpha=0.6$, time-dependent variation of approximated value of the Eq. (22)

\section{SN Applied Sciences}




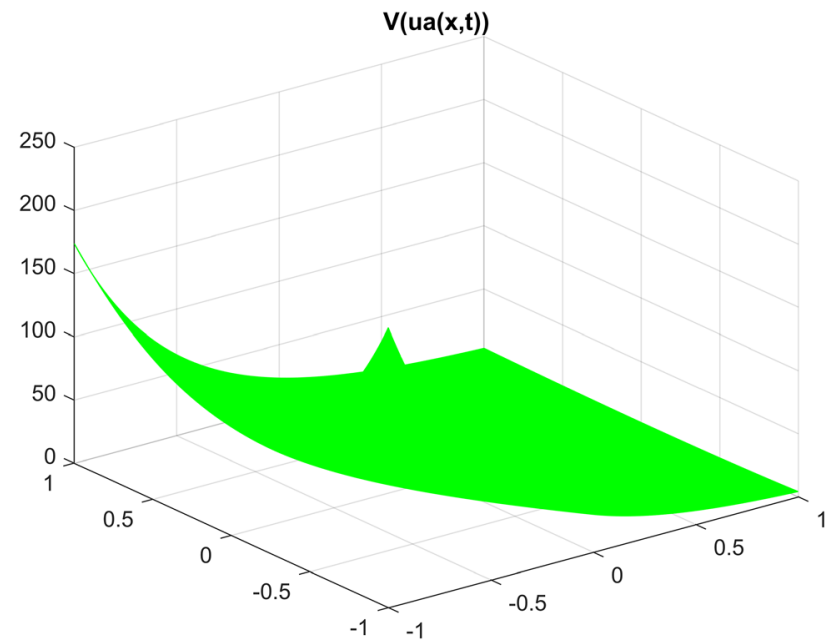

Fig. 5 For $\alpha=0.9$, time-dependent variation of variance of the Eq. (22)

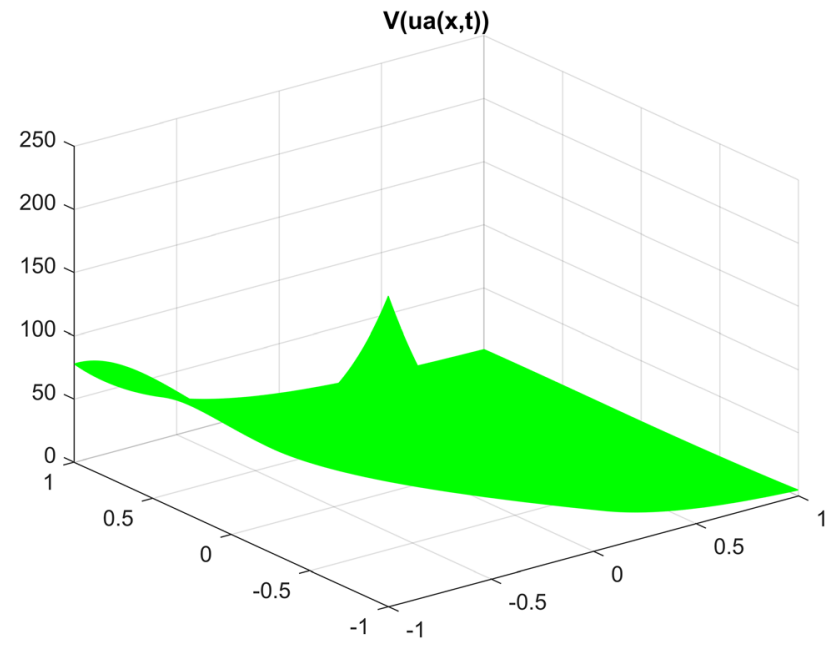

Fig. 6 For $\alpha=0.8$, time-dependent variation of variance of the Eq. (22)

Thus, the approximate solution of Eq. (28) is obtained as the following

$$
\begin{aligned}
u_{a}(x, t) & =A a^{-x}-\frac{A a^{-x} t^{\alpha} \ln a}{\Gamma(1+\alpha)}+\frac{A a^{-x} t^{2 \alpha} \ln ^{2} a}{\Gamma(1+2 \alpha)}-\frac{A a^{-x} t^{3 \alpha} \ln ^{3} a}{\Gamma(1+3 \alpha)} \\
& +\cdots+\frac{A a^{-x}\left(-\ln a t^{\alpha}\right)^{n}}{\Gamma(1+n \alpha)} \\
& =A a^{-x}\left(1-\frac{t^{\alpha} \ln a}{\Gamma(1+\alpha)}+\frac{t^{2 \alpha} \ln ^{2} a}{\Gamma(1+2 \alpha)}-\frac{t^{3 \alpha} \ln ^{3} a}{\Gamma(1+3 \alpha)}+\cdots+\frac{\left(-\ln a t^{\alpha}\right)^{n}}{\Gamma(1+n \alpha)}\right) \\
& =A a^{-x} E_{\alpha}\left[-\ln a t^{\alpha}\right] .
\end{aligned}
$$

The form $u_{a}(x, t)=A a^{-x-t}$ is the approximate solution of the Eq. (28) for $\alpha=1$. Also, this form is the exact solution of the Eq. (28) for $\alpha=1$.

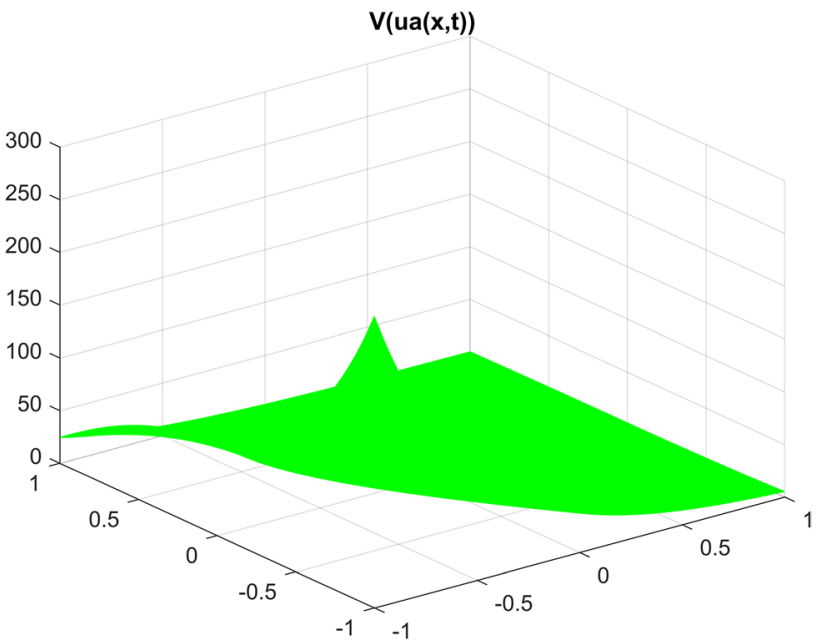

Fig. 7 For $\alpha=0.7$, time-dependent variation of variance of the Eq. (22)

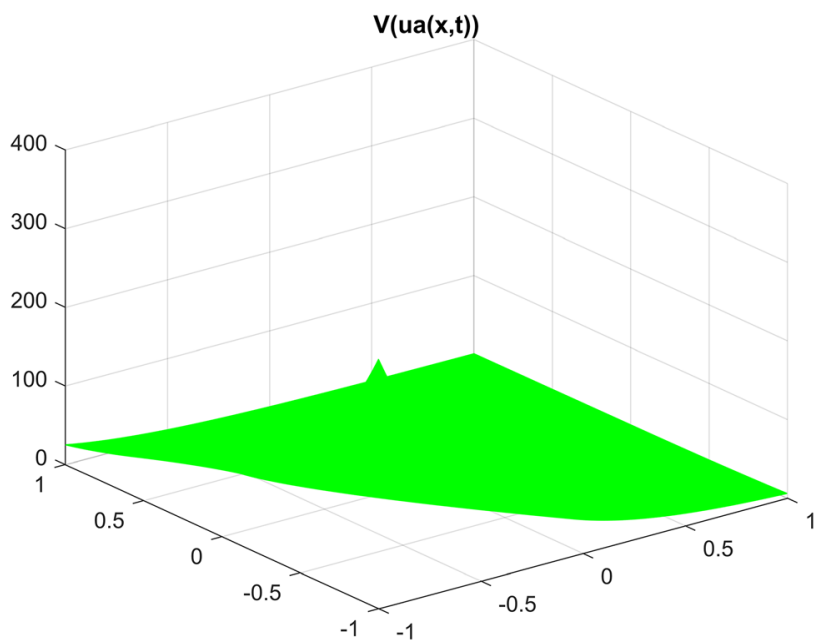

Fig. 8 For $\alpha=0.6$, time-dependent variation of variance of the Eq. (22)

In Table 4, we chose $a=1$ and $a=2$, because for $a=1$, it allows us to compare the the exact solution between approximate solution obtained with the fifth-order NSTIM and the VIM solution. $A=3$ is randomly chosen. So the approximate solution from fifth-order NSTIM indicates that it is closer to the exact solution than the approximate solution from VIM for a random $A$ in Example 2. It provided a comparison of the fifth-order NSTIM and VIM solution for $\alpha=0.9, a=2$ and $A=3$ in Table 5. 
It is observed from Table 4 that the approximate solution obtained by NSTIM is almost similar to exact solution. Table 6 shows that absolute error is negligible, because the absolute errors in Table 1 of the article of Sakar and Ergören are bigger than our absolute errors [46]. Table 6 shows the comparison of absolute error between approximate solutions acquired from different methods and exact solution different values of $x$ and $t$. It is observed from Table 4,5 and 6 that NSTIM is superior than VIM.

Let $A \sim G(\alpha=3, \beta=2)$.

Table 4 Comparison of the exact solution, approximate solution obtained with the fifth-order NSTIM and the VIM solution for $\alpha=1$, $A=3$ and $a=2$

\begin{tabular}{lllll}
\hline$x$ & $t$ & Exact sol. & NSTIM & VIM \\
\hline 0.5 & 0.2 & 1.84671662 & 1.84671659 & 1.84762679 \\
0.5 & 0.4 & 1.60766019 & 1.60765890 & 1.61470103 \\
0.5 & 0.6 & 1.39954948 & 1.39953510 & 1.42254306 \\
0.5 & 0.8 & 1.21837859 & 1.21829928 & 1.27115289 \\
0.5 & 1.0 & 1.06066017 & 1.06036316 & 1.16053050 \\
1.0 & 0.2 & 1.30582584 & 1.30582583 & 1.30646943 \\
1.0 & 0.4 & 1.13678742 & 1.13678651 & 1.14176605 \\
1.0 & 0.6 & 0.98963093 & 0.98962076 & 1.00588985 \\
1.0 & 0.8 & 0.86152376 & 0.86146768 & 0.89884083 \\
1.0 & 1.0 & 0.75000000 & 0.74978998 & 0.82061898 \\
1.5 & 0.2 & 0.92335830 & 0.92335829 & 0.92381339 \\
1.5 & 0.4 & 0.80383009 & 0.80382945 & 0.80735051 \\
1.5 & 0.6 & 0.69977474 & 0.69976755 & 0.71127153 \\
1.5 & 0.8 & 0.60918929 & 0.60914963 & 0.63557644 \\
1.5 & 1.0 & 0.53033008 & 0.53018158 & 0.58026525 \\
\hline
\end{tabular}

Table 5 Comparison of the fifth-order NSTIM and VIM solution for $\alpha=0.9, A=3$ and $a=2$

\begin{tabular}{llll}
\hline$x$ & $t$ & NSTIM & VIM \\
\hline 0.5 & 0.2 & 1.79362121 & 1.79278800 \\
0.5 & 0.4 & 1.55491049 & 1.53936543 \\
0.5 & 0.6 & 1.36085738 & 1.34137016 \\
0.5 & 0.8 & 1.19876559 & 1.19409532 \\
0.5 & 1.0 & 1.06113562 & 1.09521457 \\
1.0 & 0.2 & 1.26828172 & 1.26769255 \\
1.0 & 0.4 & 1.09948775 & 1.08849573 \\
1.0 & 0.6 & 0.96227148 & 0.94849193 \\
1.0 & 0.8 & 0.84765527 & 0.84435290 \\
1.0 & 1.0 & 0.75033619 & 0.77443365 \\
1.5 & 0.2 & 0.89681060 & 0.89639400 \\
1.5 & 0.4 & 0.77745524 & 0.76968271 \\
1.5 & 0.6 & 0.68042869 & 0.67068508 \\
1.5 & 0.8 & 0.59938279 & 0.59704766 \\
1.5 & 1.0 & 0.53056781 & 0.54760728 \\
\hline
\end{tabular}

So the approximated values of $A$ and $A^{2}$ are obtained as follows

$E(A)=3.2=6, E\left(A^{2}\right)=3.4(1+3)=48$.

The approximated value of Eq. (33) is given by

$E\left[u_{a}(x, t)\right]=E[A] a^{-x} E_{\alpha}\left[-\ln a t^{\alpha}\right]=6 a^{-x} E_{\alpha}\left[-\ln a t^{\alpha}\right]$.

The graphs of the approximated values of the Eq. (33) for different $\alpha$ values and $a=5$ are plotted in Maple software as follows (Figs. 9, 10, 11, 12)

For $A \sim G(\alpha=3, \beta=2)$, the variance of Eq. (33) is calculated as follows

$$
\begin{aligned}
V\left[u_{a}(x, t)\right] & =E\left[u^{2}(x, t)\right]-(E[u(x, t)])^{2} \\
& =E\left[A^{2}\right] a^{-2 x}\left(E_{\alpha}\left[-\ln a t^{\alpha}\right]\right)^{2}-(E[A])^{2} a^{-2 x}\left(E_{\alpha}\left[-\ln a t^{\alpha}\right]\right)^{2} \\
& =48 a^{-2 x}\left(E_{\alpha}\left[-\ln a t^{\alpha}\right]\right)^{2}-36 a^{-2 x}\left(E_{\alpha}\left[-\ln a t^{\alpha}\right]\right)^{2} \\
& =12 a^{-2 x}\left(E_{\alpha}\left[-\ln a t^{\alpha}\right]\right)^{2} .
\end{aligned}
$$

The graphs of the variances of the Eq. (33) for different $\alpha$ values and $a=5$ are plotted in Maple software as follows (Figs. 13, 14, 15, 16)

\section{Results and discussions}

It is observed from Tables 1 and 4 that the approximate solutions obtained by NSTIM for RFPDEs is almost similar to exact solutions for RFPDEs. Tables 2 and 5 show the approximate solutions obtained from NSTIM for $\alpha=0.9$ and the solutions are observed to decrease when $x$ is constant and $t$ is increased. From Tables 2 and 5 show that solutions depend on time fractional derivative. Tables 3 and 6 show that absolute errors are negligible and decrease to 0 as number of iterations increases for different values of fractional order $\alpha$ which shows that this method also works for RFPDEs. Thus, this method is reliable, because absolute errors in Table 1 of the article of Sakar and Ergören are bigger than our absolute errors [46]. Also, the graphs of the approximated values and variances are plotted for different $\alpha$ values with Maple software, and it is observed that the approximated values and variances of the solutions which obtained from NSTIM increase while the a values is decreased. It has been observed that the overall structure of the surface graphics obtained from Maple software differ for Example 1. Similarly, for Example 2, it has been observed that the overall structure of the surface graphics obtained from Maple software differ for Example 2. 
Table 6 Comparison between absolute errors when $\alpha=1, A=3$ and $a=2$, for Example 2

\begin{tabular}{|c|c|c|c|c|c|c|c|}
\hline & \multirow[t]{2}{*}{$x$} & \multicolumn{6}{|l|}{$t$} \\
\hline & & 0.00 & 0.02 & 0.04 & 0.06 & 0.08 & 0.10 \\
\hline NSTIM & 0.00 & 0.00000 & 0.00000000 & 0.00000000 & 0.00000000 & 0.00000001 & 0.00000003 \\
\hline VIM & & 0.00000 & 0.00027000 & 0.00100000 & 0.00230000 & 0.00390000 & 0.00600000 \\
\hline NSTIM & 0.02 & 0.00000 & 0.00000000 & 0.00000000 & 0.00000000 & 0.00000001 & 0.00000003 \\
\hline VIM & & 0.00000 & 0.00027000 & 0.00100000 & 0.00230000 & 0.00390000 & 0.00590000 \\
\hline NSTIM & 0.04 & 0.00000 & 0.00000000 & 0.00000000 & 0.00000000 & 0.00000001 & 0.00000003 \\
\hline VIM & & 0.00000 & 0.00027000 & 0.00100000 & 0.00220000 & 0.00380000 & 0.00580000 \\
\hline NSTIM & 0.06 & 0.00000 & 0.00000000 & 0.00000000 & 0.00000000 & 0.00000001 & 0.00000003 \\
\hline VIM & & 0.00000 & 0.00026000 & 0.00100000 & 0.00220000 & 0.00380000 & 0.00570000 \\
\hline NSTIM & 0.08 & 0.00000 & 0.00000000 & 0.00000000 & 0.00000000 & 0.00000001 & 0.00000003 \\
\hline VIM & & 0.00000 & 0.00026000 & 0.00100000 & 0.00220000 & 0.00370000 & 0.00560000 \\
\hline NSTIM & 0.10 & 0.00000 & 0.00000000 & 0.00000000 & 0.00000000 & 0.00000001 & 0.00000003 \\
\hline VIM & & 0.00000 & 0.00025000 & 0.00100000 & 0.00210000 & 0.00370000 & 0.00560000 \\
\hline
\end{tabular}

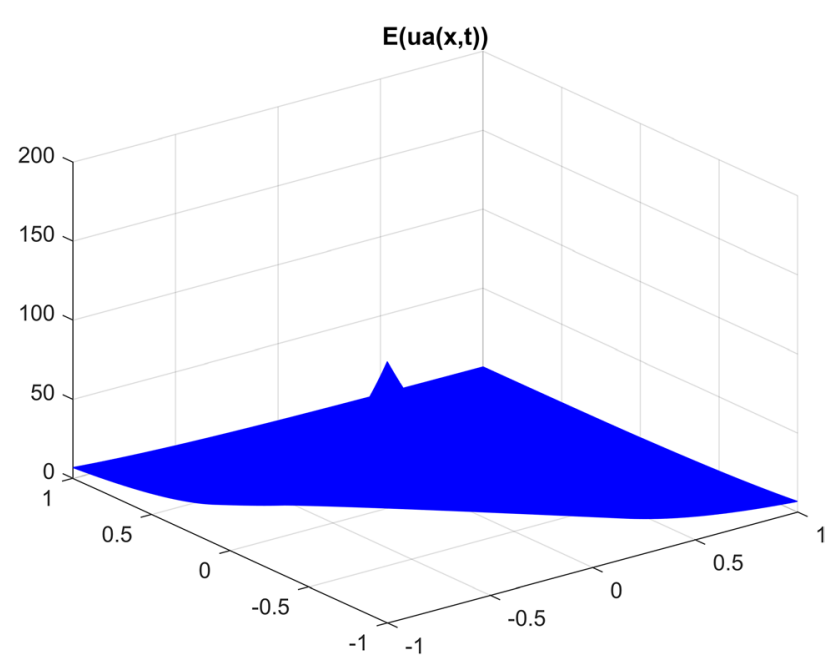

Fig. 9 For $\alpha=0.9$ and $a=5$, time-dependent variation of approximated value of the Eq. (28)

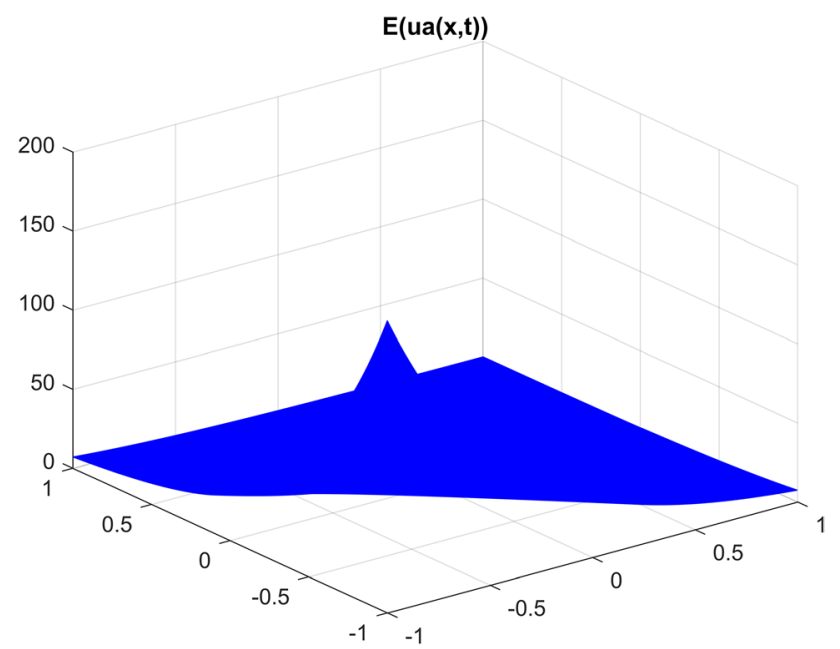

Fig. 10 For $\alpha=0.8$ and $a=5$, time-dependent variation of approximated value of the Eq. (28)

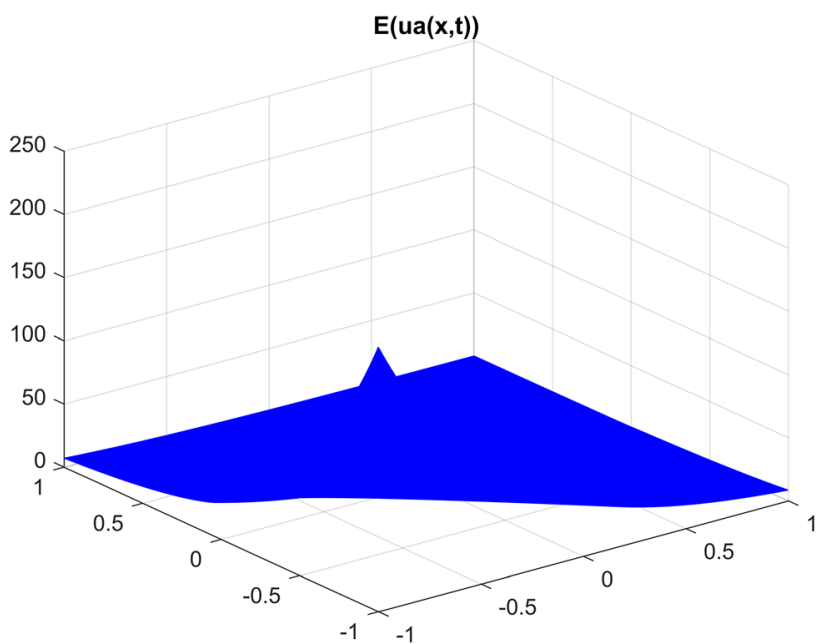

Fig. 11 For $\alpha=0.7$ and $a=5$, time-dependent variation of approximated value of the Eq. (28)

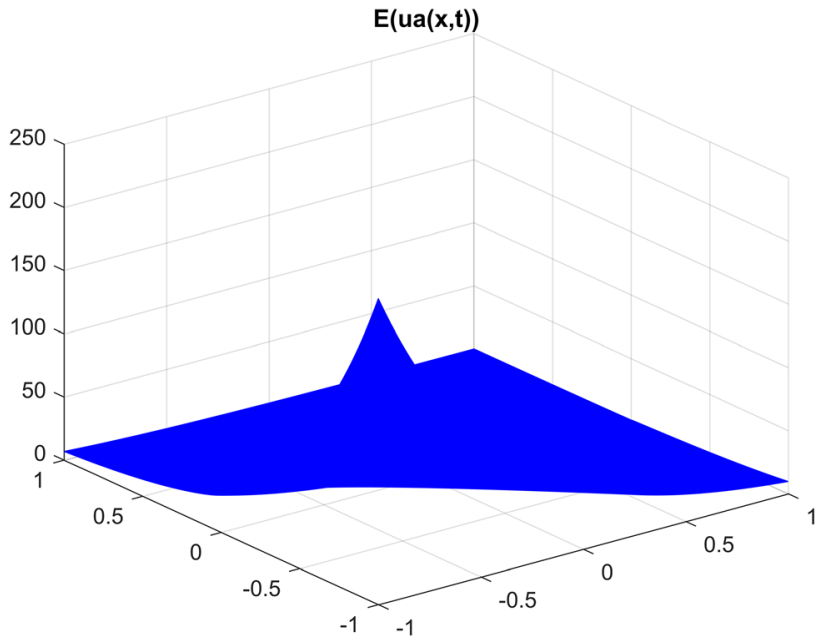

Fig. 12 For $\alpha=0.6$ and $a=5$, time-dependent variation of approximated value of the Eq. (28) 


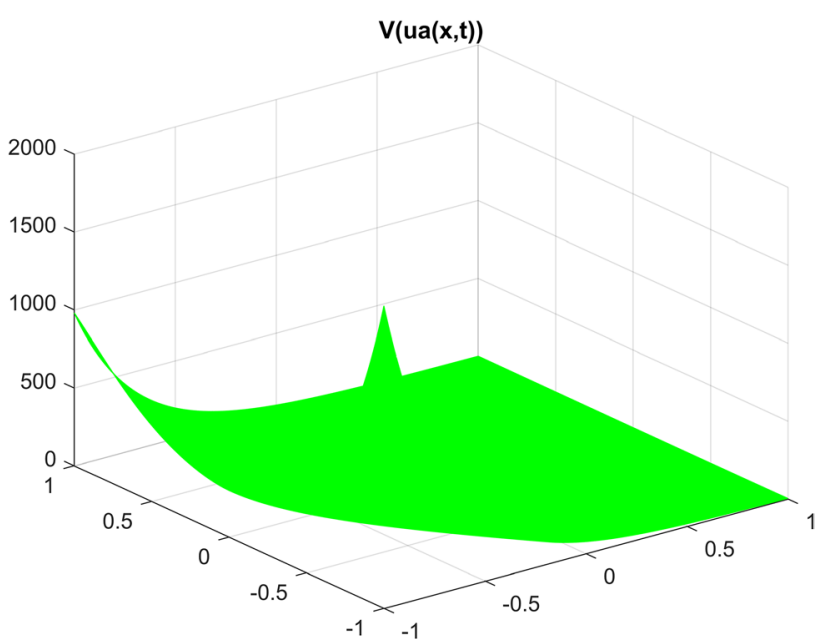

Fig. 13 For $\alpha=0.9$, time-dependent variation of variance of the Eq. (28)

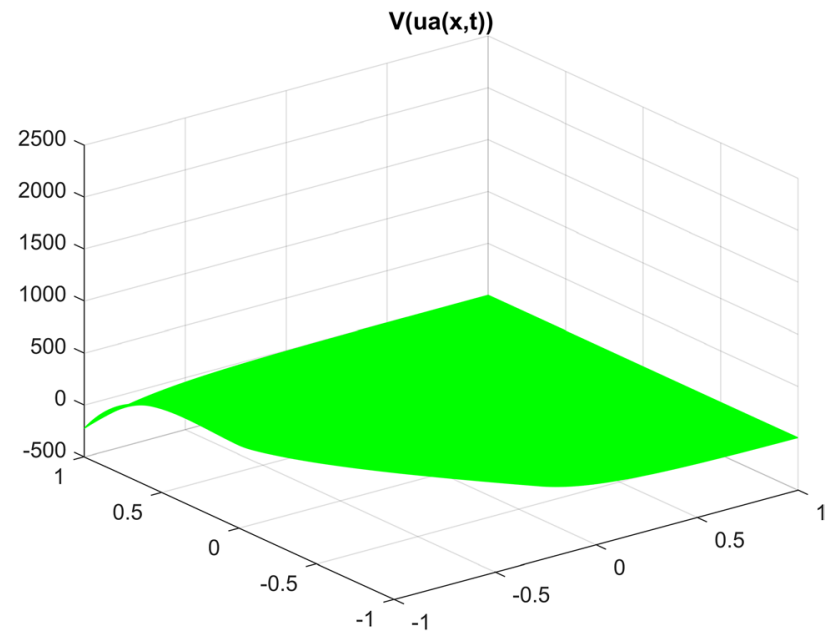

Fig. 14 For $\alpha=0.8$, time-dependent variation of variance of the Eq. (28)

\section{Conclusion}

In this study, RFPDEs are analyzed by NSTIM method. The approximate analytical solution of RFPDEs has been quickly and successfully obtained. NSTIM is more powerful than VIM as shown in the absolute error comparison in Tables 3 and 6 . Thus, it is concluded that NSTIM is reliable, effective and powerful in getting the approximate solutions for different classes of random component linear and nonlinear fractional of ordinary and partial differential equations.

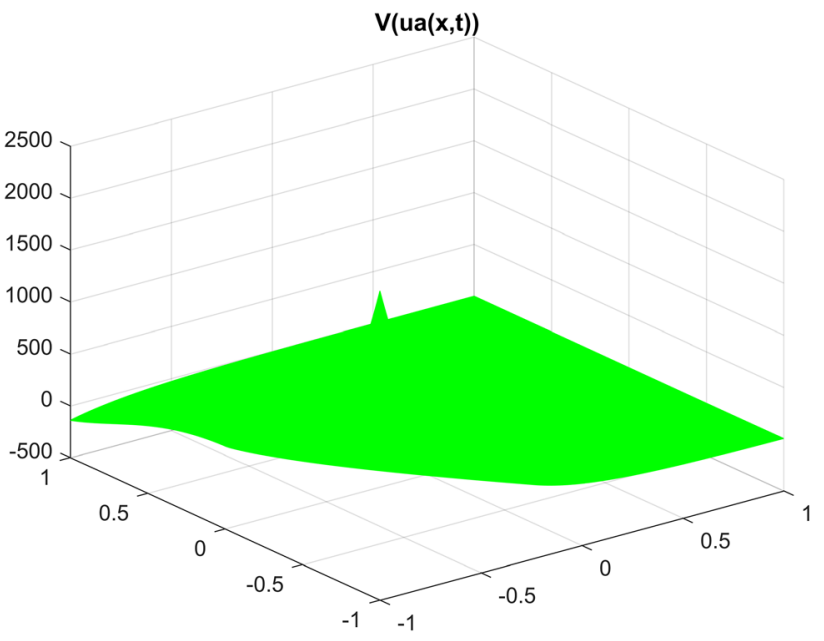

Fig. 15 For $\alpha=0.7$, time-dependent variation of variance of the Eq. (28)

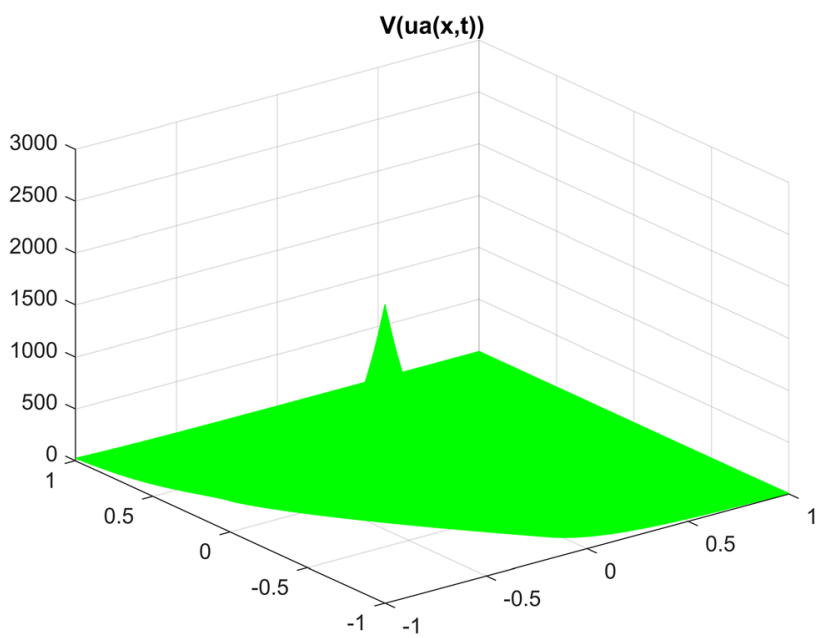

Fig. 16 For $\alpha=0.6$, time-dependent variation of variance of the Eq. (28)

\section{Compliance with ethical standards}

Conflict of interest The authors declare that they have no conflict of interest.

\section{References}

1. Hilfer R (2000) Application of fractional calculus in physics. World Scientific Publishing Company, Singapore

2. Kilbas A, Srivastava H, Trujillo J (2006) Theory and applications of fractional differential equations. Elsevier, Amsterdam

3. Kumar M, Daftardar-Gejji V (2019) Exact solutions of fractional partial differential equations by Sumudu transform iterative 
method. In: Daftardar-Gejji V (ed) Fractional calculus and fractional differential equations. Birkhäuser, Singapore, pp 157-180

4. Miller KS, Ross B (1993) An introduction to the fractional calculus and fractional differantial equations. Wiley, New York

5. Oldham KB, Spanier J (1974) The fractional calculus. Academic Press, New York

6. Podlubny I (1999) Fractional differential equations. Academic Press, San Diego

7. Samko SG, Kilbas AA, Marichev OI (1993) Fractional ıntegrals and derivatives theory and applications. Gordon and Breach, New York

8. Abu-Gdairi R, Al-Smadi M, Gumah G (2015) An expansion iterative technique for handling fractional differential equations using fractional power series scheme. J Math Stat 11(2):29-38

9. Baleanu D, Golmankhaneh AK, Baleanu MC (2009) Fractional electromagnetic equations using fractional forms. Int J Theor Phys 48(11):3114-3123

10. Baleanu D, Jajarmi A, Hajipour M (2018) On the nonlinear dynamical systems within the generalized fractional derivatives with Mittag-Leffler kernel. Nonlinear Dyn 2018:1-18

11. Baleanu D, Asad JH, Jajarmi A (2018) New aspects of the motion of a particle in a circular cavity. Proc Rom Acad Ser A 19(2018):361-367

12. Baleanu D, Jajarmi A, Bonyah E, Hajipour M (2018) New aspects of poor nutrition in the life cycle within the fractional calculus. Adv Differ Equ 1:230

13. Jajarmi A, Baleanu D (2018) Suboptimal control of fractionalorder dynamic systems with delay argument. J Vib Control 24(12):2430-2446

14. Jajarmi A, Baleanu D (2018) A new fractional analysis on the interaction of HIV with CD4 + T-cells. Chaos, Solitons Fractals 113(2018):221-229

15. Khalil H, Al-Smadi M, Moaddy K, Khan RA, Hashim I (2016) Toward the approximate solution of fractional order nonlinear mixed derivative and nonlocal boundary value problems. Discrete Dyn Nat Soc. https://doi.org/10.1155/2016/5601821

16. Klimek K (2005) Fractional sequential mechanics-models with symmetric fractional derivative. Czechoslov J Phys 55:1447-1453

17. Laskin N (2000) Fractional quantum mechanics. Phys Rev E 62:3135-3145

18. Mainardi $F$ (2010) Fractional calculus and waves in linear viscoelasticity. Imperial College Press, London

19. Wazwaz AM (1999) A reliable modification of Adomian decomposition method. Appl Math Comput 102:77-86

20. He JH (1999) Homotopy perturbation technique. Comput Methods Appl Mech Engrgy 178(3):257-262

21. Yüzbaşı Ş (2013) A numerical approximation for Volterra's population growth model with fractional order. Appl Math Model 37(5):3216-3227

22. Yüzbaşı Ş (2013) Numerical solutions of fractional Riccati type differential equations by means of the Bernstein polynomials. Appl Math Comput 219(11):6328-6343

23. Yüzbaşı Ş (2016) Numerical solutions of hyperbolic telegraph equation by using the Bessel functions of first kind and residual correction. Appl Math Comput 287:83-93

24. Yüzbaşı Ş (2016) A collocation method for numerical solutions of fractional-order logistic population model. Int J Biomath 9(02):1650031

25. Yüzbaşı Ş (2017) A numerical method for solving secondorder linear partial differential equations under Dirichlet, Neumann and Robin boundary conditions. Int J Comput Methods 14(02):1750015
26. Yüzbaşı Ş (2018) A collocation approach for solving two-dimensional second-order linear hyperbolic equations. Appl Math Comput 338:101-114

27. Yüzbaşı Ş, Karaçayır M (2018) A Galerkin-type method to solve one-dimensional telegraph equation using collocation points in Initial and boundary conditions. Int J Comput Methods 15(05):1850031

28. Merdan M, Gökdoğan A, Yıldırım A, Mohyud-din ST (2012) Numerical simulation of fractional Fornberg-Whitham equation by differential transformation method. Abst Appl Anal. https:// doi.org/10.1155/2012/965367

29. He JH (1999) Variational iteration method-a kind of non-linear analytical technique: some examples. Int J Non-Linear Mech 34(4):699-708

30. Wu GC, Lee EVM (2010) Fractional variational iteration method and its application. Phys Lett A 374:2506-2509

31. Cortés JC, Jódar L, Villafuerte L, Villanueva RJ (2007) Computing mean square approximations of random diffusion models with source term. Math Comput Simul 76(1-3):44-48

32. El-Tawil M, Sohaly MA (2012) Mean square convergent three points finite difference scheme for random partial differential equations. J Egypt Math Soc 20(3):188-204

33. Wang K, Liu S (2016) A new Sumudu transform iterative method for time-fractional Cauchy reaction-diffusion equation. Springer Plus 5(1):865

34. Watugala GK (1993) Sumudu transform: a new integral transform to solve differential equations and control engineering problems. Int J Math Educ Sci Technol 24(1):35-43

35. Weerakoon S (1994) Application of Sumudu transform to partial differential equations. Int J Math Educ Sci Technol 25(2):277-283

36. Weerakoon S (1998) Complex inversion formula for Sumudu transform. Int J Math Edu Sci Technol 29(4):618-621

37. Demiray ST, Bulut H, Belgacem FBM (2015) Sumudu transform method for analytical solutions of fractional type ordinary differential equations. Math Prob Eng. https://doi. org/10.1155/2015/131690

38. Kumar M, Daftardar-Gejji V (2018) Exact solutions of fractional partial differential equations by Sumudu transform iterative method. arXiv:1806.03057v1

39. Rahman NAA, Ahmad MZ (2017) Solving fuzzy fractional differential equations using fuzzy Sumudu transform. J Nonlinear Sci Appl 10(5):2620-2632

40. Prakash A, Kumar M, Baleanu D (2018) A new iterative technique for a fractional model of nonlinear Zakharov-Kuznetsov equations via Sumudu transform. Appl Math Comput 334:30-40

41. Dimovski IH (1990) Convolutional calculus. Kluwer Academic Publishers, Amsterdam

42. Yang XJ, Baleanu D, Srivastava HM (2016) Local fractional integral transforms and their applications. Academic Press, London

43. Chaurasia VBL, Singh J (2010) Application of Sumudu transform in Schrödinger equation occurring in quantum mechanics. Appl Math Sci 4(57):2843-2850

44. Roussas GG (2014) Introduction to probability, 2nd edn. Academic Press, Amsterdam

45. Daftardar-Gejji V, Jafari H (2006) An iterative method for solving nonlinear functional equations. J Math Anal Appl 316:753-763

46. Sakar MG, Ergören H (2015) Alternative variational iteration method for solving the time-fractional Fornberg-Whitham equation. Appl Math Model 39(14):3972-3979

Publisher's Note Springer Nature remains neutral with regard to jurisdictional claims in published maps and institutional affiliations. 